\title{
Lifelong Clinical Impact of the Presence of Sarcomere Gene Mutation in Japanese Patients With Hypertrophic Cardiomyopathy
}

\author{
Yasuteru Nakashima, MD; Toru Kubo, MD; Kenta Sugiura, MD; Yuri Ochi, MD; \\ Asa Takahashi, MD; Yuichi Baba, MD; Takayoshi Hirota, MD; Naohito Yamasaki, MD; \\ Akinori Kimura, MD; Yoshinori L. Doi, MD; Hiroaki Kitaoka, MD
}

\begin{abstract}
Background: Hypertrophic cardiomyopathy (HCM) is mainly caused by mutations in sarcomere genes. Regarding the clinical implications of genetic information, little is known about the lifelong clinical effect of sarcomere mutations in Japanese HCM patients.

Methods and Results: We studied 211 consecutive Japanese patients with HCM who had agreed to genetic testing between 2003 and 2013. Genetic analyses were performed by direct DNA sequencing in the 6 common sarcomere genes (MYH7, MYBPC3, TNNT2, TNNI3, TPM1, ACTC). Through variant filtering, 21 mutations were identified in 67 patients. After excluding 8 patients whose

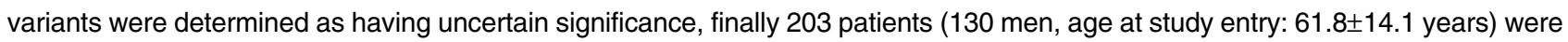
investigated for clinical presentation and course. At the time of study entry, patients with mutations were younger, had more frequent non-sustained ventricular tachycardia, had greater interventricular wall thickness, were more frequently in the dilated phase and less frequently had apical HCM. Through their lifetimes, a total of $98 \mathrm{HCM}$-related morbid events occurred in 72 patients. Survival analysis revealed that patients with sarcomere gene mutations experienced those morbid events significantly more frequently, and this tendency was more prominent for lethal arrhythmic events.
\end{abstract}

Conclusions: In our HCM cohort, patients with sarcomere gene mutations had poorer lifelong outcome. Genetic information is considered important for better management of $\mathrm{HCM}$.

Key Words: Hypertrophic cardiomyopathy; Sarcomere mutations

H ypertrophic cardiomyopathy $(\mathrm{HCM})$ is a primary myocardial disease with heterogeneous morphological, functional, and clinical features. HCM is the most common hereditary cardiomyopathy in the world, with a prevalence of up to 1 in 500 people. $^{1-3}$ It is an autosomal-dominant trait disease that is mainly caused by mutation in the genes encoding sarcomere proteins. Since the pioneering research by Seidman and colleagues in $1990,{ }^{4}$ several studies had revealed the existence of sarcomere gene mutations in $40-60 \%$ of HCM patients. ${ }^{2,3}$ Genetic testing of HCM patients is recommended in several guidelines to facilitate identification of first-degree family members, because first-degree relatives are considered to be at risk for developing HCM.1,2 However, because the clinical effect of sarcomere gene mutations is controversial in patients with HCM-positive phenotype, we conducted this study to clarify the relationship between the presence of these mutations and lifelong clinical outcomes in Japanese patients with HCM.

Methods
Subjects
This study was a single-center retrospective study. We
studied 211 consecutive Japanese patients with HCM who
had agreed to genetic testing between March 2003 and
December 2013. The study entry of the patients was the time
of DNA collection for genetic testing. All patients were
evaluated at Kochi Medical School Hospital for confirming
of diagnosis, risk assessment, and symptom management.
The diagnosis of HCM was based on echocardiographic
demonstration of unexplained left ventricular hypertrophy
(LVH: maximum LV wall thickness $\geq 15$ mm) in the absence
of other cardiac diseases that could produce hypertrophy of
such magnitude (e.g., arterial hypertension, aortic stenosis

Received January 16, 2020; revised manuscript received June 5, 2020; accepted June 22, 2020; J-STAGE Advance Publication released online August 22, 2020 Time for primary review: 33 days

Department of Cardiology and Geriatrics, Kochi Medical School, Kochi University, Nankoku (Y.N., T.K., K.S., Y.O., A.T., Y.B., T.H., N.Y., Y.L.D., H.K.); Department of Molecular Pathogenesis, Medical Research Institute, Tokyo Medical and Dental University, Tokyo (A.K.), Japan

Mailing address: Toru Kubo, MD, Department of Cardiology and Geriatrics, Kochi Medical School, Kochi University, Oko-cho, Nankoku 783-8505, Japan. E-mail: jm-kubotoru@kochi-u.ac.jp

All rights are reserved to the Japanese Circulation Society. For permissions, please e-mail: cj@j-circ.or.jp

ISSN-1346-9843 


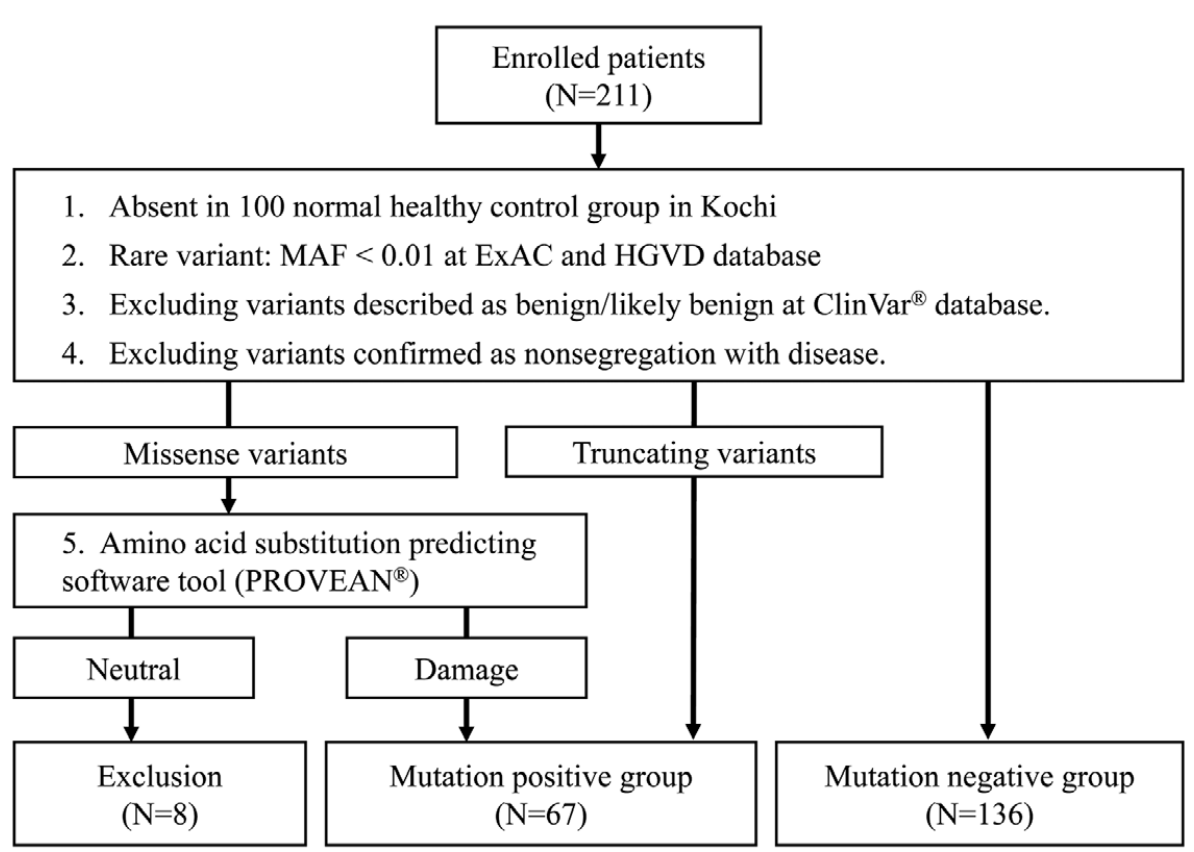

Figure 1. Flowchart of variant filtering. ExAC, Exome Aggregation Consortium browser; HGVD, Human Genetic Variation Database; MAF, minor allele frequency.

or storage disease). The study was approved by the Ethics Committee on Medical Research of Kochi Medical School, and followed both the Declaration of Helsinki and the ethical standards of the responsible committee on human experimentation. Written informed consent was given by all of the patients or their parents in accordance with the guidelines of the Ethics Committee on Medical Research of Kochi Medical School. Patients' data, including medical history, laboratory data, 12-lead ECG, and M-mode, 2-D and Doppler echocardiography, were collected from their medical records. In echocardiographic examination, echocardiographic findings including severity of LVH and HCM subtypes were assessed by previously reported methods and definitions. 5

Survival and clinical data were collected during serial clinical visits. The study closed on December 31, 2017. Regarding clinical outcomes, we focused on the lifelong outcome from birth to last follow-up. The following 3 modes of HCM-related morbid events were used for survival analysis: (1) arrhythmic events, including sudden cardiac death (SCD), successful recovery from ventricular tachycardia (VT) or ventricular fibrillation (VF), and appropriate implantable cardioverter-defibrillator (ICD) discharge, with SCD being defined as sudden and unexpected death after collapse in the absence of or $<1 \mathrm{~h}$ from the onset of symptoms in patients who previously experienced a relatively stable or uneventful clinical course; (2) heart failure (HF) events including $\mathrm{HF}$ death and $\mathrm{HF}$ hospitalization, with HF death being defined as the death of patients with progressive cardiac decompensation $\leq 1$ year before death, particularly if complicated by pulmonary edema or evolution to the dilated phase defined as an ejection fraction (modified Simpson's method) $<50 \%$; and (3) embolic events, including stroke-related death, non- fatal stroke, and non-fatal embolism, with stroke-related death being defined as death that occurred as a result of probable or proven embolic stroke.

\section{Genetic Analysis}

In this investigation, we screened for mutations in the protein-coding exons of 6 sarcomere genes encoding cardiac myosin-binding protein $\mathrm{C}$ ( $M Y B P C 3)$, cardiac $\beta$ myosin heavy chain $(M Y H 7)$, cardiac troponin T (TNNT2), cardiac troponin I (TNNI3), $\alpha$ tropomyosin (TPMI), and $\alpha$ actin $(A C T C)$. After written informed consent was given, peripheral blood samples were taken and the DNA was extracted using a purification kit (no. 51140; Qiagen Inc., Hilden, Germany). In vitro amplification of all exons was performed by polymerase chain reaction using oligonucleotide primers. Information on primer sequences are shown in Supplementary Table 1. Sequencing was performed by using a BigDye Terminator Cycle Sequencing Kit (no. 4336774; Applied Biosystems Inc., Foster City, CA, USA). The sequences were analyzed on an ABI PRISM 3100Avant Genetic Analyzer in accordance with the manufacturer's manual. If a mutation was identified, confirmation was obtained by re-analysis with direct sequencing from a second blood sample.

\section{Genetic Diagnosis}

After the genetic analysis, we conducted variant filtering based on the flowchart shown in Figure 1. First, we selected variants that were absent in a normal healthy control group of 100 subjects in Kochi Prefecture. Second, because the homozygous variant was not detected in our genetic analysis, we selected rare variants defined as variants with a minor allele frequency $<0.01$ for autosomal-dominant heterozygous variants in genetic databases, including the 


\begin{tabular}{|c|c|c|c|c|}
\hline Gene & Nucleotide change & Amino acid change & Variant type & $\begin{array}{l}\text { Pathogenicity according } \\
\text { to } 2015 \text { ACMG guidelines }\end{array}$ \\
\hline MYВРC3 & c.318delT & p.Pro106fs & Frameshift & Pathogenic \\
\hline MYBPC3 & c. $478 \mathrm{C}>\mathrm{T}$ & p.Arg160Trp & Missense & Uncertain significance \\
\hline МYBPCЗ & c. $890 \mathrm{C}>\mathrm{G}$ & p.Ser297Ter & Stop gained & Pathogenic \\
\hline МYBPCЗ & c. $1112 \mathrm{C}>\mathrm{T}$ & p.Pro371Leu & Missense & Likely pathogenic \\
\hline MYВРCЗ & c. $1136 \mathrm{~A}>\mathrm{C}$ & p.His379Pro & Missense & Uncertain significance \\
\hline МYBРCЗ & c.1597delC & p.Gln533fs & Frameshift & Pathogenic \\
\hline МYBPCЗ & c.1777delT & p.Ser593fs & Frameshift & Pathogenic \\
\hline МYВРСЗ & c. $2285 \mathrm{~T}>\mathrm{A}$ & p.Val762Asp & Missense & Likely pathogenic \\
\hline МYВРСЗ & c. $2459 \mathrm{G}>\mathrm{A}$ & p.Arg820Gln & Missense & Pathogenic \\
\hline МYBPCЗ & c.2833_2834delCG & p.Arg945fs & Frameshift & Pathogenic \\
\hline MYBPC3 & c. $3412 C>T$ & p.Arg1138Cys & Missense & Likely pathogenic \\
\hline MYH7 & c. $727 \mathrm{C}>\mathrm{T}$ & p.Arg243Cys & Missense & Likely pathogenic \\
\hline MYH7 & c. $1357 \mathrm{C}>\mathrm{T}$ & p.Arg453Cys & Missense & Pathogenic \\
\hline MYH7 & c. $1686 C>A$ & p.Asn562Lys & Missense & Likely pathogenic \\
\hline MYH7 & c. $1987 \mathrm{C}>\mathrm{T}$ & p.Arg663Cys & Missense & Pathogenic \\
\hline MYH7 & c. $2605 \mathrm{C}>\mathrm{T}$ & p.Arg869Cys & Missense & Likely pathogenic \\
\hline MYH7 & c. $2803 \mathrm{G}>\mathrm{A}$ & p.Glu935Lys & Missense & Likely pathogenic \\
\hline MYH7 & c. $4259 \mathrm{G}>\mathrm{A}$ & p.Arg1420Gln & Missense & Likely pathogenic \\
\hline TPM1 & c. $662 A>G$ & p.Tyr221Cys & Missense & Uncertain significance \\
\hline TNNI3 & c. $484 \mathrm{C}>\mathrm{T}$ & p.Arg162Trp & Missense & Pathogenic \\
\hline ACTC1 & c. $293 \mathrm{~T}>\mathrm{C}$ & p.Val98Ala & Missense & Uncertain significance \\
\hline
\end{tabular}

ACMG, American College of Medical Genetics and Genomics; ACTC, $a$ actin gene; MYBPC3, cardiac myosin-binding protein $\mathrm{C}$ gene; $M Y H 7$, cardiac $\beta$ myosin heavy chain gene; TNNI3, cardiac troponin I gene; TPM1, a tropomyosin gene.

Exome Aggregation Consortium (ExAC) browser (http:// exac.broadinstitute.org) and the Human Genetic Variation Database (HGVD) (http://www.hgvd.genome.med.kyotou.ac.jp). Next, we excluded variants that were described as "benign" or "likely benign" in an online database of human genotypes and phenotypes (ClinVar; http://www.ncbi.nlm. nih.gov/clinvar/). We also excluded variants that were confirmed as non-segregation with the disease in the family. We then defined truncating variants, such as nonsense or frameshift variants, as pathogenic. If these variants were missense variants, we checked them through online amino acid substitution predicting software (protein variant effect analyzer (PROVEAN ${ }^{\circledR}$ ); http://provean.jcvi.org/index.php). Finally, we concluded that only the missense variants that were judged as "damage" in PROVEAN were pathogenic mutations, and those that were judged as "neutral" were excluded from our study as variants of uncertain significance. In addition to our method of using variant filtering regarding the genetic diagnosis, we also present our results based on the interpretation of sequence variants recommended by the 2015 American College of Medical Genetics and Genomics (ACMG) standards and guidelines. ${ }^{6}$

\section{Statistical Analysis}

All data are expressed as mean \pm SD or frequency (percentage). Comparisons of clinical characteristics between mutation-positive and mutation-negative groups were assessed by Student's t-test for normally distributed variables. Pearson's chi-square test and t-test were used for comparisons between categorical and continuous variables, and Fisher's exact test was used when the expected frequency was $<5$. Event-free estimates curves were calculated by the Kaplan-Meier method, and the log-rank test was used for comparison between groups. The multivariate
Cox proportional hazards model was used to analyze the relationship between HCM-related morbid events and clinical factors, mainly including genetic or familial parameters (family history of HCM was finally not included because there was a strong correlation between sarcomere gene mutation and family history of HCM). All calculations were performed using $\mathrm{JMP}^{\circledR}$ 12.0.1 software (SAS institute Inc., Cary, NC, USA).

\section{Results}

In total, 21 mutations were identified in 57 probands among $67(33 \%)$ of 203 study patients after excluding 8 patients who had variants determined as being of uncertain significance (Figure 1). The identified mutations are summarized in Table 1, which also shows pathogenicity classified according to the 2015 ACMG guidelines ${ }^{6}$ (detailed information of the variants shown in Supplementary Table 2). Among the 55 patients with a proven family history of HCM, prevalence of sarcomere gene mutations was up to $71 \%$ (39 of 55 familial HCM patients). Conversely, the remaining 148 patients who were determined as having non-familial HCM included $28(19 \%)$ patients with sarcomere gene mutations.

\section{Clinical Characteristics}

Patients were divided into 2 groups based on the presence of sarcomere gene mutations: mutation-positive $(n=67)$ and mutation-negative $(n=136)$. Their clinical characteristics are shown in Table 2 (clinical characteristics of the 2 groups classified by the 2015 ACMG guidelines shown in Supplementary Table 3). At baseline, mutation-positive patients were significantly younger than mutation-negative patients both at the time of study entry $(56.7 \pm 15.6$ years vs. $64.2 \pm 12.6$ years, $\mathrm{P}=0.001)$ and at the time of diagnosis of 


\begin{tabular}{|c|c|c|c|c|}
\hline & $\begin{array}{l}\text { Overall } \\
(n=203)\end{array}$ & $\begin{array}{c}\text { Mutation } \\
\text { positive } \\
(n=67)\end{array}$ & $\begin{array}{c}\text { Mutation } \\
\text { negative } \\
(n=136)\end{array}$ & $P$ value \\
\hline \multicolumn{5}{|l|}{ Clinical characteristics } \\
\hline Age at study entry (DNA collection) (years) & $61.5 \pm 14.2$ & $56.7 \pm 15.6$ & $64.2 \pm 12.6$ & 0.001 \\
\hline Age at initial diagnosis (years) & $55.2 \pm 15.8$ & $50.2 \pm 16.7$ & $58.3 \pm 14.5$ & 0.001 \\
\hline Sex male, $\mathrm{n}(\%)$ & $130(64)$ & $36(54)$ & $94(69)$ & 0.04 \\
\hline NYHA class III/IV at study entry, n (\%) & $10(5)$ & $5(7)$ & $5(4)$ & 0.30 \\
\hline Family history of $\mathrm{HCM}, \mathrm{n}(\%)$ & $55(27)$ & $39(58)$ & $16(12)$ & $<0.0001$ \\
\hline AF at study entry, $n(\%)$ & $53(27)$ & $17(26)$ & $36(27)$ & 1.00 \\
\hline Reason for diagnosis & & & & 0.08 \\
\hline Cardiac symptoms, n (\%) & $103(51)$ & $35(52)$ & $68(50)$ & \\
\hline Incidental, n (\%) & $93(45)$ & $27(40)$ & $66(49)$ & \\
\hline Family screening, $\mathrm{n}(\%)$ & $7(4)$ & $5(8)$ & $2(1)$ & \\
\hline \multicolumn{5}{|l|}{ Risk of SCD } \\
\hline Family history of SCD, $\mathrm{n}(\%)$ & $33(16)$ & $17(25)$ & $16(12)$ & 0.02 \\
\hline Wall thickness $\geq 30 \mathrm{~mm}$ at study entry, $\mathrm{n}(\%)$ & $2(1)$ & $2(3)$ & $0(0)$ & 0.11 \\
\hline Rest LVOTO $\geq 30 \mathrm{mmHg}$ at study entry, $\mathrm{n}(\%)$ & $27(13)$ & $7(10)$ & $20(15)$ & 0.51 \\
\hline Past history of syncope, $\mathrm{n}(\%)$ & $21(10)$ & $10(15)$ & $11(8)$ & 0.15 \\
\hline Past history of NSVT, n (\%) & $27(13)$ & $14(21)$ & $13(10)$ & 0.03 \\
\hline \multicolumn{5}{|l|}{ Echocardiographic findings at study entry } \\
\hline Maximum wall thickness $(\mathrm{mm})$ & $19.8 \pm 3.8$ & $19.8 \pm 4.2$ & $19.7 \pm 3.7$ & 0.91 \\
\hline Interventricular wall thickness (mm) & $15.3 \pm 4.1$ & $16.6 \pm 4.6$ & $14.6 \pm 3.8$ & 0.003 \\
\hline Posterior wall thickness $(\mathrm{mm})$ & $11.0 \pm 2.0$ & $10.6 \pm 2.0$ & $11.2 \pm 1.9$ & 0.04 \\
\hline LV end-diastolic diameter (mm) & $45.9 \pm 6.1$ & $45.4 \pm 7.1$ & $46.0 \pm 5.5$ & 0.52 \\
\hline LV end-systolic diameter (mm) & $27.2 \pm 6.7$ & $27.6 \pm 8.5$ & $26.9 \pm 5.7$ & 0.51 \\
\hline Fractional shortening (\%) & $41.4 \pm 8.8$ & $40.1 \pm 10.5$ & $42.1 \pm 7.7$ & 0.16 \\
\hline LA diameter $(\mathrm{mm})$ & $44.5 \pm 7.2$ & $45.3 \pm 8.0$ & $44.2 \pm 6.7$ & 0.31 \\
\hline Rest LVOTO $\geq 30 \mathrm{mmHg}, \mathrm{n}(\%)$ & $27(13)$ & $7(10)$ & $20(15)$ & 0.51 \\
\hline LV morphology & & & & $<0.0001$ \\
\hline Dilated phase of HCM, n (\%) & $10(5)$ & $7(10)$ & $3(2)$ & \\
\hline HOCM, n (\%) & $30(15)$ & $7(10)$ & $23(17)$ & \\
\hline Midventricular obstruction, n (\%) & $9(4)$ & $1(1)$ & $8(6)$ & \\
\hline Apical HCM, n (\%) & $39(19)$ & $3(4)$ & $36(26)$ & \\
\hline Non-obstructive HCM, n (\%) & $115(57)$ & $49(73)$ & $66(49)$ & \\
\hline
\end{tabular}

HCM, hypertrophic cardiomyopathy; HOCM, Hypertrophic obstructive cardiomyopathy; LVOTO, left ventricular outflow tract obstruction; NYHA, New York Heart Association; NSVT, non-sustained ventricular tachycardia; SCD, sudden cardiac death.

$\operatorname{HCM}(50.2 \pm 16.7$ years vs. $58.3 \pm 14.5$ years, $\mathrm{P}=0.001)$. The proportion of patients with a positive family history of HCM was higher in the mutation-positive group than in the mutation-negative $(58 \%$ vs. $12 \%, \mathrm{P}<0.0001)$, and the proportion of male patients was significantly smaller in the mutation-positive group ( $54 \%$ vs. $69 \%, \mathrm{P}=0.04)$. Regarding the risk of SCD, a larger proportion of patients in the mutation-positive group had a positive family history of SCD $(25 \%$ vs. $12 \%, \mathrm{P}=0.02)$ and a past history of nonsustained VT (NSVT) ( $21 \%$ vs. $10 \%, \mathrm{P}=0.03)$. Among the echocardiographic parameters, mutation-positive patients showed a greater thickness of the interventricular wall (16.6 \pm 4.6 vs. $14.6 \pm 3.8 \mathrm{~mm}, \mathrm{P}=0.003)$, but there were no significant differences between groups for $\mathrm{LV}$ end-diastolic diameter, LV end-systolic diameter, maximum LV wall thickness, fractional shortening, left atrial diameter and ratio of LV outflow tract obstruction. Regarding LV morphology, the proportion of patients in the dilated phase of HCM was larger in the mutation-positive group (10\% vs. $2 \%$ ). Conversely, the proportion of apical HCM patients was larger in the mutation-negative group ( $26 \%$ vs. $4 \%$ ). The prevalence of sarcomere gene mutations in apical HCM patients was as low as $8 \%$, but was $39 \%$ in nonapical HCM patients. There was a significant difference between these groups $(\mathrm{P}<0.0001$, log-rank test) (Figure 2).

\section{Clinical Outcomes}

Figure 3 is a time line showing the study patients' ages and the HCM-related morbid events through their lifetimes. A total of 72 patients ( $35 \%$ of the study patients) experienced a HCM-related morbid event; 21 patients died from HCMrelated morbid events during the follow-up period after study entry.

Results of lifelong survival analyses are shown in Figure 4. Kaplan-Meier curves for composite events including arrhythmic events, HF events and embolic events revealed that patients in the mutation-positive group experienced significantly more HCM-related morbid events than did patients in the mutation-negative group $(\mathrm{P}<0.0001, \log$ rank test; Figure 4A). This tendency was more prominent 

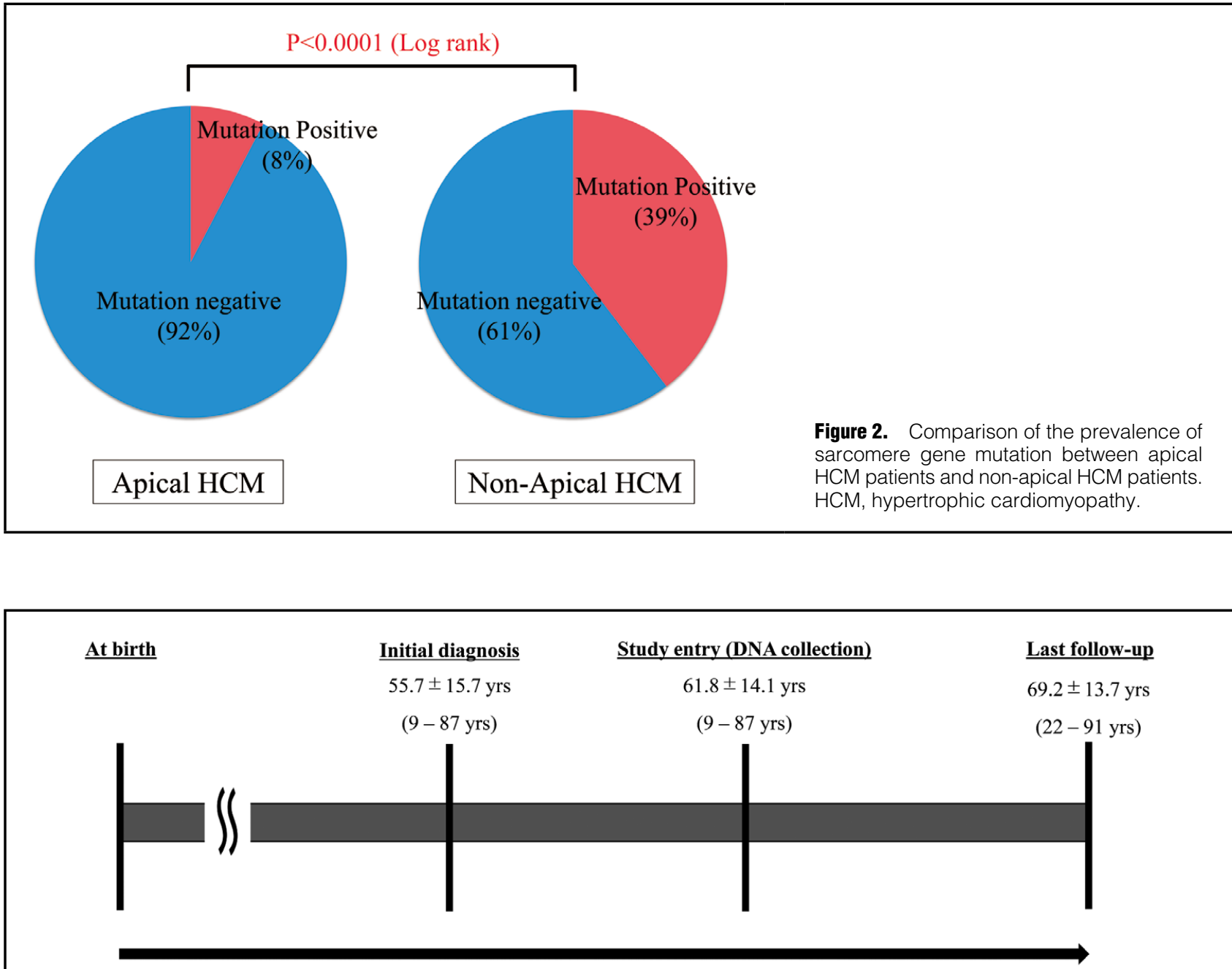

Through the lifetime

98 HCM-related morbid events in 72 patients

Figure 3. Time line with patient ages in this study and HCM-related morbid events through their lifetime. HCM, hypertrophic cardiomyopathy.

for arrhythmic events, and mutation-negative patients rarely experienced fatal arrhythmic events in their lifetimes (Figure 4B). Furthermore, mutation-positive patients experienced significantly more $\mathrm{HF}$ events than did mutationnegative patients, although this tendency was relatively modest compared with the tendency for arrhythmic events (Figure 4C). On the other hand, there was no significant difference between the 2 groups for embolic events (Figure 4D). Similar results for clinical outcomes were observed when the patients were divided into 2 groups by interpretation of sequence variants based on the 2015 ACMG guidelines (Supplementary Figure).

In addition, a multivariate Cox proportional hazards model showed that the presence of sarcomere mutation as well as younger age at initial diagnosis were independent predictors of HCM-related morbid events through the patient's lifetime (Table 3). We divided the study patients into 2 groups according to proven family history (familial
HCM patients $(n=55)$ and non-familial HCM patients $(n=148)$ ), and examined the effect sarcomere gene mutations on prognosis in each group. There was no significant difference between the mutation-positive and mutation-negative groups among the familial HCM patients (Figure 5A), but among the non-familial HCM patients, the mutationpositive group experienced significantly more HCM-related morbid events than did the mutation-negative patients (Figure 5B).

\section{Discussion}

This study is the first to show the prevalence of sarcomere gene mutations and the clinical characteristics of patients with sarcomere gene mutations in a Japanese rural regional HCM cohort. This study also showed that Japanese HCM patients with sarcomere gene mutations had more frequent HCM-related morbid events including arrhythmic events, 

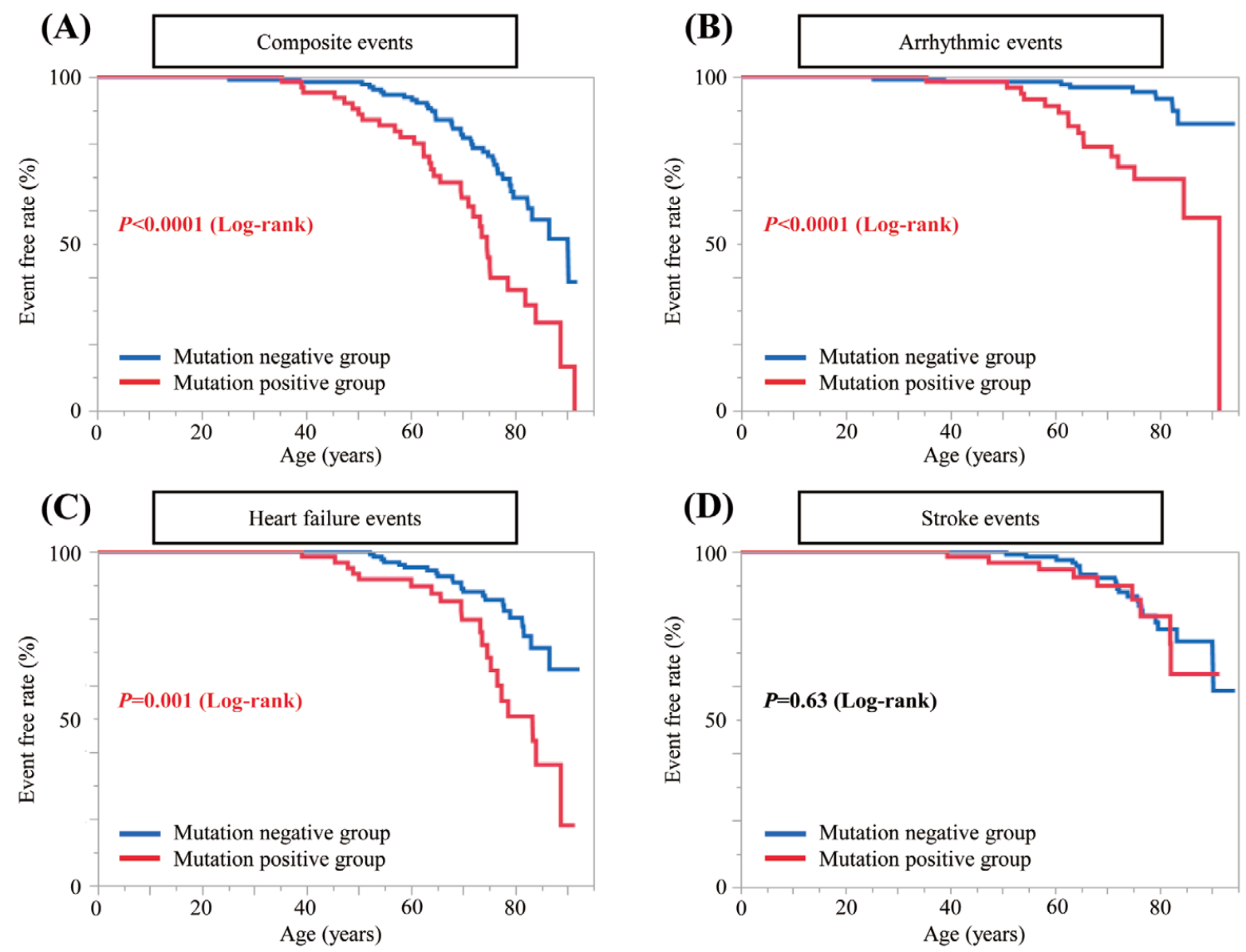

Figure 4. Kaplan-Meier curves for the incidence of HCM-related morbid events through a patient's lifetime. (A) Composite events including arrhythmic events, heart failure events, and embolic events. (B) Arrhythmic events including sudden cardiac death, successful recovery from VT or VF, and appropriate ICD discharge. (C) Heart failure events including heart failure death and heart failure hospitalization. (D) Embolic events including embolic stroke and systemic embolism. HCM, hypertrophic cardiomyopathy; ICD, implantable cardioverter-defibrillator; VF, ventricular fibrillation; VT, ventricular tachycardia.

HF events and embolic events than did HCM patients without sarcomere gene mutations through their lifetime. To the best of our knowledge, this study is the first study showing the clinical significance of sarcomere gene mutation as a lifelong risk predictor in Japanese adult HCM patients.

In our HCM cohort, the proportion of patients with positive sarcomere gene mutations was 33\% (67 of 203 patients), which is relatively low in comparison with the proportion in past studies. ${ }^{7-14}$ The 2 main possible reasons are as follows. (1) Our study cohort consisted of local residents of Kochi Prefecture, so the proportion of elderly people was relatively high and the proportion of young patients with a family history of cardiomyopathy was relatively low compared with proportions in previously reported groups of referral centers. As a result, the prevalence of gene mutations in our cohort was relatively low. (2) In our cohort, approximately $20 \%$ of the patients were classified as having apical HCM in terms of LV morphology, as shown in Table 2. Apical HCM is a more common subtype of HCM in Asians and is characterized by a low prevalence of familial HCM. Hence, in our cohort the rate of positive gene mutations was lower than in past studies. There are few reports showing the prevalence of sarcomere gene mutations among Japanese HCM patients, including those with apical HCM. In that sense, the data obtained in our study are unique.

In our cohort, HCM patients with sarcomere gene mutations experienced significantly more HCM-related morbid events through their lifetime. Our study showed that HCM patients with sarcomere gene mutations experienced HCMrelated morbid events from the age of 40 years and that the incidence of events was relatively high compared with that in mutation-negative patients. In a previous study, Olivotto and colleagues ${ }^{\mathbf{1 0}}$ revealed that HCM patients with sarcomere gene mutations had an increased risk of combined endpoints of cardiovascular death, non-fatal stroke, and progression to advanced HF. They also reported that mutation-positive HCM patients had a greater probability of LV systolic and diastolic dysfunction. Other studies have shown almost the same tendency, ${ }^{10,15-17}$ which is consistent with the results of our study. However, there has not been a study in which the clinical significance of gene mutations, especially in terms of cardiovascular events such as arrhythmic events, $\mathrm{HF}$ events and embolic events, was examined in detail. In 
Table 3. Univariate and Multivariate Analyses for HCM-Related Morbid Events

\begin{tabular}{lccccc} 
& \multicolumn{2}{c}{ Univariate analysis } & & \multicolumn{2}{c}{ Multivariate analysis } \\
\cline { 2 - 3 } \cline { 5 - 6 } Sex (male) & $\mathbf{H R}(\mathbf{9 5 \%} \mathbf{C l})$ & $\mathbf{P}$ value & & HR (95\% Cl) & P value \\
Sarcomere gene mutation & $1.05(0.65-1.72)$ & 0.84 & & $0.87(0.53-1.44)$ & 0.59 \\
Family history of SCD & $2.53(1.58-4.04)$ & $<0.0001$ & & $2.08(1.27-3.37)$ & 0.003 \\
Age at initial diagnosis (1 year) & $1.21(0.62-2.17)$ & 0.56 & & $1.02(0.52-1.84)$ & 0.96 \\
\cline { 5 - 6 } & $0.93(0.91-0.95)$ & $<0.0001$ & & $0.93(0.91-0.95)$ & $<0.0001$ \\
\hline
\end{tabular}

$\mathrm{Cl}$, confidence interval; HCM, hypertrophic cardiomyopathy; HR, hazard ratio; SCD, sudden cardiac death.
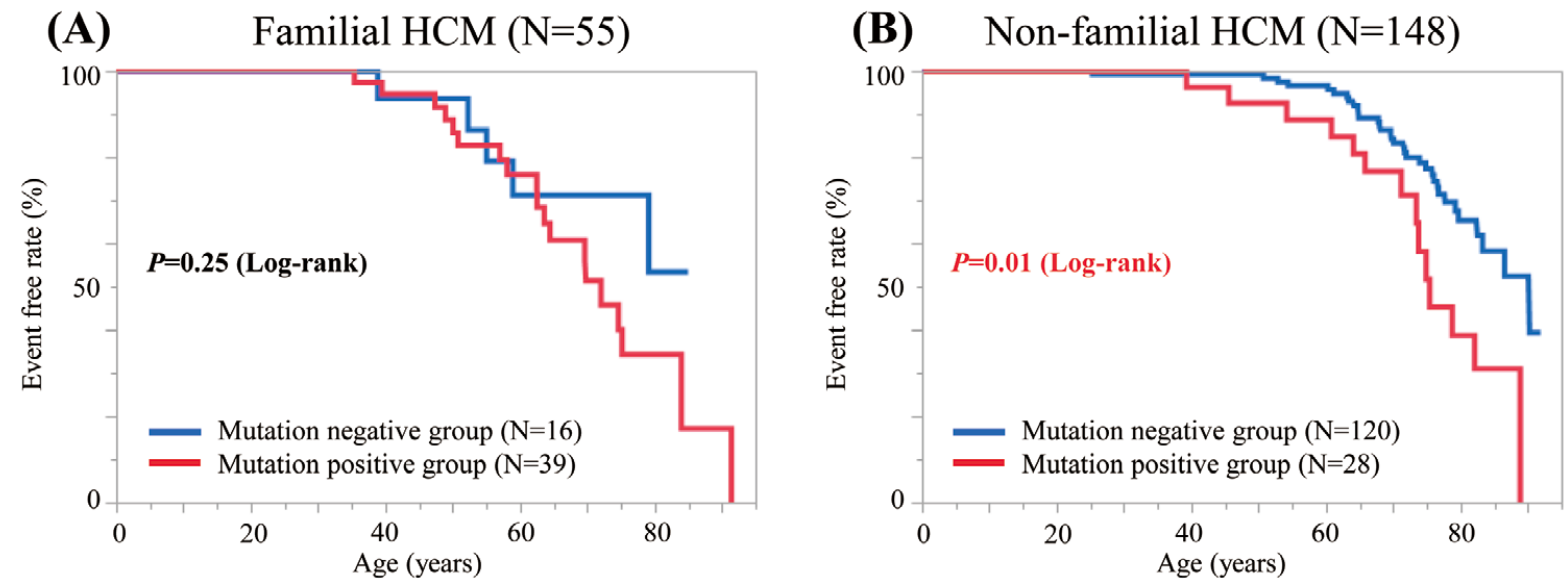

Figure 5. Kaplan-Meier curves for the incidence of HCM-related morbid events through a patient's lifetime. Composite events including arrhythmic events, heart failure events, and embolic events among HCM patients with (A) proven family history and (B) without proven family history. HCM, hypertrophic cardiomyopathy.

our study, the tendency for deterioration in the prognosis of HCM patients with sarcomere gene mutations was more prominent for arrhythmic events, including SCD, successful recovery from VT or VF, and appropriate ICD discharge. Therefore, our study suggests that more consideration should be given to implantation of a cardioverter-defibrillator for HCM patients with sarcomere gene mutations.

The precise reason for such prognostic differences is unclear, but coronary microvascular dysfunction may be involved. Olivotto and colleagues ${ }^{\mathbf{1 8}}$ reported a relationship between sarcomere gene mutation and microvascular dysfunction. They investigated microvascular function in HCM patients by using ${ }^{13} \mathrm{~N}$-labeled ammonia positron emission tomography, which revealed that mutation-positive patients had substantially lower microvascular blood flow than mutation-negative patients. That result suggests that sarcomere gene mutations may be related to more prevalent and severe impairment of coronary microcirculation, ultimately causing myocardial fibrosis and systolic dysfunction. However, the link between sarcomere gene mutation and coronary microvascular dysfunction has still not been investigated in detail. One hypothesis is that precursor cells of the coronary artery are exposed to mechanical stress from abnormal myocardial cells during the embryonic stage, resulting in vascular endothelial cell dysfunction. ${ }^{19}$ However, there are few findings supporting such a hypothesis, and further investigations are needed.

Concerning clinical usefulness, the results of our study suggest that confirmation of genetic mutations may be useful for predicting complications accompanying HCM over the patient's lifetime, especially prediction of arrhythmic events including SCD. Furthermore, data from our investigation suggest that family history screening without genetic testing may be insufficient to predict HCM-related morbid events. In our cohort, 148 patients were determined to be non-familial HCM based on medical interview and nongenetic screening test. However, genetic testing revealed that $28(19 \%)$ of these patients had sarcomere gene mutations. Moreover, these 28 patients experienced significantly more HCM-related morbid events than the patients without genetic mutation (Figure 5B). That result suggests that in addition to non-genetic tests such as medical interviews, screening for sarcomere gene mutations may be useful for predicting the prognosis of HCM patients.

\section{Study Limitation}

Genetic examination was conducted by direct sequencing targeting protein-coding exons of 6 sarcomere genes. Whole-exon sequencing or whole-genome sequencing using a next-generation sequencer is necessary in future studies.

\section{Conclusions}

In our HCM cohort, patients with sarcomere gene mutations had a poorer lifelong outcome than did patients without mutations, and this tendency was more prominent for 
arrhythmic events. The results suggest that the presence of a sarcomere gene mutation is a useful predictor for HCMrelated morbid events, and genetic information is considered to be important for better management of HCM.

\section{IRB Information}

Approved by the Ethics Committee on Medical Research of Kochi Medical School, reference number ERB-002361.

\section{Disclosures}

Conflicts of Interest: None declared. H.K. is a member of Circulation Journal' Editorial Team.

\section{Grants}

This work was supported, in part, by Grant-in-Aid for Scientific Research from the Japan Society for the Promotion of Science (16K09440) (to T. Kubo).

\section{References}

1. Gersh BJ, Maron BJ, Bonow RO, Dearani JA, Fifer MA, Link MS, et al. 2011 ACCF/AHA guideline for the diagnosis and treatment of hypertrophic cardiomyopathy: A report of the American College of Cardiology Foundation/American Heart Association Task Force on Practice Guidelines. Circulation 2011; 124: e783-e 831

2. Elliott PM, Anastasakis A, Borger MA, Borggrefe M, Cecchi F, Charron P, et al. 2014 ESC guidelines on diagnosis and management of hypertrophic cardiomyopathy: The Task Force for the Diagnosis and Management of Hypertrophic Cardiomyopathy of the European Society of Cardiology (ESC). Eur Heart J 2014; 35: $2733-2779$

3. Maron BJ, Maron MS, Semsarian C. Genetics of hypertrophic cardiomyopathy after 20 years: Clinical perspectives. $\mathrm{J}$ Am Coll Cardiol 2012; 60: 705-715.

4. Geisterfer-Lowrance AA, Kass S, Tanigawa G, Vosberg HP, McKenna W, Seidman CE, et al. A molecular basis for familial hypertrophic cardiomyopathy: A beta cardiac myosin heavy chain gene missense mutation. Cell 1990; 62: 999-1006.

5. Kubo T, Hirota T, Baba Y, Ochi Y, Takahashi A, Yamasaki N, et al. Patients' characteristics and clinical course of hypertrophic cardiomyopathy in a regional Japanese cohort: Results from Kochi RYOMA Study. Circ J 2018; 82: 824-830.

6. Richards S, Aziz N, Bale S, Bick D, Das S, Gastier-Foster J, et al. Standards and guidelines for the interpretation of sequence variants: A joint consensus recommendation of the American College of Medical Genetics and Genomics and the Association for Molecular Pathology. Genet Med 2015; 17: 405-424.

7. Erdmann J, Daehmlow S, Wischke S, Senyuva M, Werner U, Raible J. Mutation spectrum in a large cohort of unrelated con- secutive patients with hypertrophic cardiomyopathy. Clin Genet 2003; 4: 339-349.

8. Mörner S, Richard P, Kazzam E, Hellman U, Hainque B, Schwartz K, et al. Identification of the genotypes causing hypertrophic cardiomyopathy in northern Sweden. J Mol Cell Cardiol 2003; 35: 841-849.

9. Richard P, Charron P, Carrier L, Ledeuil C, Cheav T, Pichereau $\mathrm{C}$, et al. Hypertrophic cardiomyopathy: Distribution of disease genes, spectrum of mutations, and implications for a molecular diagnosis strategy. Circulation 2003; 107: 2227-2232.

10. Olivotto I, Girolami F, Ackerman MJ, Nistri S, Bos JM, Zachara $\mathrm{E}$, et al. Myofilament protein gene mutation screening and outcome of patients with hypertrophic cardiomyopathy. Mayo Clin Proc 2008; 83: 630-638.

11. Millat G, Bouvagnet P, Chevalier P, Dauphin C, Jouk PS, Da Costa A, et al. Prevalence and spectrum of mutations in a cohort of 192 unrelated patients with hypertrophic cardiomyopathy. Eur J Med Genet 2010; 53: 261 - 267.

12. Brito D, Miltenberger-Miltenyi G, Vale Pereira S, Silva D, Diogo AN, Madeira H. Sarcomeric hypertrophic cardiomyopathy: Genetic profile in a Portuguese population. Rev Port Cardiol 2012; 31: 577-587.

13. Otsuka H, Arimura T, Abe T, Kawai H, Aizawa Y, Kubo T, et al. Prevalence and distribution of sarcomeric gene mutations in Japanese patients with familial hypertrophic cardiomyopathy. Circ J 2012; 76: 453-461.

14. Liu W, Liu W, Hu D, Zhu T, Ma Z, Yang J, et al. Mutation spectrum in a large cohort of unrelated Chinese patients with hypertrophic cardiomyopathy. Am J Cardiol 2013; 112: 585-589.

15. Li Q, Gruner C, Chan RH, Care M, Siminovitch K, Williams L, et al. Genotype-positive status in patients with hypertrophic cardiomyopathy is associated with higher rates of heart failure events. Circ Cardiovasc Genet 2014; 7: 416-422.

16. Ingles J, Burns C, Bagnall RD, Lam L, Yeates L, Sarina T, et al Nonfamilial hypertrophic cardiomyopathy: Prevalence, natural history, and clinical implications. Circ Cardiovasc Genet 2017; 10: $\mathrm{e} 001620$

17. Ho CY, Day SM, Ashley EA, Michels M, Pereira AC, Jacoby D et al. Genotype and lifetime burden of disease in hypertrophic cardiomyopathy: Insights from the Sarcomeric Human Cardiomyopathy Registry (SHaRe). Circulation 2018; 138: 1387-1398.

18. Olivotto I, Girolami F, Sciagrà R, Ackerman MJ, Sotgia B, Bos $\mathrm{JM}$, et al. Microvascular function is selectively impaired in patients with hypertrophic cardiomyopathy and sarcomere myofilament gene mutations. J Am Coll Cardiol 2011; 58: 839-848.

19. Olivotto I, Cecchi F, Poggesi C, Yacoub MH. Developmental origins of hypertrophic cardiomyopathy phenotypes: A unifying hypothesis. Nat Rev Cardiol 2009; 6: 317-321.

\section{Supplementary Files}

Please find supplementary file(s);

http://dx.doi.org/10.1253/circj.CJ-20-0027 